\title{
Isolation and Identification of Bacteria Associated with Balangu (Roasted Meat Product) Sold in Bauchi. Nigeria
}

\author{
Moshood A. Yusuf ${ }^{1}$, Tengku Haziyamin Abdul Tengku Abdul Hamid ${ }^{2}$, Ibrahim \\ Hussain $^{3}$ \\ ${ }^{1,2}$ Department of Biotechnology, Kulliyyah of Science, International Islamic University Malaysia, Bandar \\ InderaMahkota, Jalan Istana, 25200 Kuantan, Malaysia. \\ ${ }^{3}$ Biology programme, Gombe state university NIGERIA.
}

\begin{abstract}
A total of twenty (20) "balangu" samples (roasted meat product) were collected from five (5) different retail outlet namely: Yelwa, Government Reserve Area, Bayangari, Railway and Mudalawal Motor Park within Bauchi metropolis; Ten (10) samples were spiced and ten (10) others were unspiced. Bacteriological analysis was carried out on the samples for isolation and identification of bacteria associated with such meat products as well total aerobic plate count. Fourteen (14) specie of bacteria which are of public health importance were isolated and identified. The percentage of occurrences of bacteria isolates was highest for Bacillus cereus with $19.6 \%, 12.5 \%$ for Staphylococcus aureus, $10.7 \%$ for Escherichia coli, 7.1\% for Bacillus alvis, Proteus mirabilis and Streptococcus faecalis, then $5.4 \%$ for both Proteus vulgaris and Shigella sp, hence Bacillus alvis, Proteus mirabilis, Salmonella sp, Citrobacter freundii, Klebsiella pneumonia and Enterococcus eurogenase shows the lowest occurrence with 3.5\%. Also the mean aerobic plate count (cfu/g) for unspicedbalangu samples was $2.25 \times 10^{6}, 2.05 \times 10^{6}, 2.47 \times 10^{6}, 2.79 \times 10^{6}$, $2.78 \times 10^{6}$ while that of spiced balangu samples was $2.66 \times 10^{6}, 2.36 \times 10^{6}, 2.69 \times 10^{6}, 2.85 \times 10^{6}, 2.89 \times 10^{6}$ for Yelwa, Government Reserve Area, Bayangari, Railway and Mudalawal motor park retail outlet respectively.
\end{abstract}

Keywords:- Roasted meat, bacteriological analysis, aerobic count,and bacteriological analysis,balangu, spiced

\section{INTRODUCTION}

Meat is an edible animal flesh which comprises principally the muscular tissue, and also includes internal organ called viscera such as heart, liver, kidney, intestine and bladder. [1].[2] relate that the bulk of meat is derived from goat, cattle, pig, sheep, and poultry.

According to [3] the chemical composition of meat varies considerably with age, species, degree of fatness of animal, the part of carcass involved etc.

Because of the enormous value of meat in the diet, there exist large markets for meet and meat products worldwide at varying money value hence their demands increase day by day across the globe.

Meat products are obtained when raw meat or preserved meat (cured meat) are altered in form by grinding, pressing, drying and other processes then augmented in flavour by smoking, spicing or blending with other food. These meat products are subjected to combination of several basic processing steps before reaching their final form. Therefore meat products are also termed as processed meat.Micro-organisms that occur in meat and meat products most times are responsible for food borne illness. These micro-organisms are Bacillus $s p$, Clostridium sp, Escherichia coli, Salmonella sp, Shigella sp, Staphylococcus aureus, Streptococcus pyogenes, Proteus, Pseudomonas, Leuconostoc, Lactobacillus sp, Micrococcus, Mycobacterium sp, Vibrio sp etc. [4].Salmonella may be transferred from raw meat to cooked meat by hands, surfaces or utensils [5].

There exist different types of meat product ranging from the industrially processed corned beef, ham, bacon sausage to the indigenous Nigeria traditionally processed ready-to-eat meat product such as "balangu" (roasted meat), "kilishi, dan-bu-nama, tsire, jirga, ndako, banda, suya and many more". [6]. Other meat products includes "beef kebab" in Europe, "kyiskiyma" in central Africa, and "sogo djemine" in Mali.

Prepation of "Balangu" involves using boneless flesh, offals and viscera of mutton or beef. The collected boneless raw meat is washed with clean water cut and sliced into a thick sheet of average thickness of $0.9 \mathrm{~cm}-1.5 \mathrm{~cm}$ then further wide-spread on a clean table. The sliced meat uniformly dusted with little salt and maggi spices is then placed on an iron net skewer and roasted over a smokeless fire for about 40-60 minute with regular turning and sprinkling of peanut oil to increase the tenderness while smoking and to make the meat products looks very attractive to customers. 
Isolation and Identification of Bacteria Associated with Balangu (Roasted Meat Product)...

The distance of the stick to the fire is normally $35 \mathrm{~cm}-40 \mathrm{~cm}$ and at temperature of approximately $92^{\circ} \mathrm{C}$, but variation occurs between latter or former depending on certain factors like quantity and time factor.

The production and consumption of ready-to-eat roasted meat product cut across religion, social status and gender, and of the entire meat product mentioned, "'balangu"' is the most popular, easily assessable within Bauchi metropolis.One of the sole problems in the delivery of suya is the poor product sanitation and retail which significantly influence its bacteria quality. Hence this factor is a strong negative reason against its consumption particularly by some educated individuals for they insist on the product being hot at the time purchase.The factor which affects the microbial growth in both raw meat and meat products are intrinsic factors which includes: moisture content, $\mathrm{pH}$, nutritive value and absence of barriers or inhibitory substance to the growth of micro-organism.

Extrinsic factors such as temperature, relative humidity, $\mathrm{O}_{2}$ availability and other factors including chemical and physical properties of food(meat) affect microbial growth [1].

Meanwhile the processing of meat products includes those prepared product for consumption, increases the quality, texture, flavour, colour as well as reduce bacterial contaminant of meat used [7].On heating, frying or smoking, meat losses its moisture which results in increasing the concentration of other nutrients thus protein and fats are present in larger amount per unit weight in dried meat samples than in their fresh forms. However excessive heat leads to appreciable loss in biological values as protein are denatured and essential amino acid are made unavailable by thermally induced chemical reactions and loss of water soluble vitamins (affect vitamin content).

In Nigeria and most developing African countries, salting and hot smoking are the common traditional method widely used in meat preservation thus lots of meat products are obtained by salting, spicing and smokedrying (hot smoking). Higher temperature are usually applied in hot smoking process where the intention is to partly cook and dry the product as well as impact a smoke flavour to it.

In tropical countries such as Nigeria, hot smoking is a method of choice as seen in various meat product such as "balangu", tsire, dan-bu-nama, while excessive drying or hard drying for total expulsion of moisture is often necessary to preserve the product for a longer period of time as seen in kilishi (meat product).

As a result of manner of processing and handling of various meat products it is exposed to lots of microorganisms among which are Salmonella sp., Shigella sp., Vibriosp., Campylobacter sp., Bacillus cereus, and other related fecal coliform(bacteria). Aspergillus, Penicilium, Cladosporium, Alternaria, Mucor (fungi).

This work aid to study the level of bacterial contamination in "balangu" sold within Bauchi metropolis and therefore determines its safety for human consumption. Hence the isolation and identification of bacteria present in meat and its product is necessary to anticipate the extent of spoilage under storage and handling procedures [8].

\subsection{Sample collection}

\section{MATERIAL AND METHODS}

A total number of twenty (20) "balangu" samples were purchased from five (5) different retail outlets which comprises ofYelwa, Government Reserve Area, Bayangari, Railway and Mudalawal Motor Parkwithin Bauchi metropolis. The samples were collected in a clean dry and sterile polythene bags and transported to the laboratory for bacteriological analysis or refrigerated at $4^{0} \mathrm{c}$ till analysis was carried out on the collected sample.

\subsection{Criteria for sample collection}

Below are the criteria used for collection of twenty (20) "balangu" samples for the research work.

I. Two (2) types of "balangu" samples were collected for the research work namely: The spiced and unspiced "balangu" samples.

II. A number of four (4) "balangu" samples were collected from each of the five (5) retail outletlisted above.

III. Two (2) samples of prepared and unspiced "balangu" (roasted meat product) samples were collected in a clean and dry sterile polythene bag.

IV. Also two (2) samples of prepared and spiced "balangu" meat product were collected in a clean dry and sterile polythene bag.

Therefore four (4) "balangu" samples were collected from each of the five (5) retail outlet summing up to twenty (20) "balangu" samples to be used for the bacteriological analysis.

\subsection{Sample preparation}

The "balangu" sample was aseptically cut into thin smaller pieces using sterile knife. It was thoroughly blended using sterile blender. 1 gram from the blended sample was then placed in $10 \mathrm{ml}$ of distilled water and mixed thoroughly to give a good homogenate used as stock. 
Isolation and Identification of Bacteria Associated with Balangu (Roasted Meat Product)...

Several dilutions were achieved up to 5 fold $\left(10^{-5}\right)$ for each prepared samples using $1 \mathrm{ml}$ from stock homogenate and $9 \mathrm{ml}$ of sterile distilled water for the serial dilution experiment. This was carried out in order to obtain discrete colony.

\subsection{Plating and culture on media}

$0.1 \mathrm{ml}$ was taken from 1-5 folds dilution and dispensed in sterile Petri dishes by spread plate method thus was allowed to set firmly for (5) minutes afterward inoculated plates were incubated at $37^{0} \mathrm{c}$ for 24 hours.

\subsection{Microscopy and colonial identification}

Characterization and identification of the colony isolates was achieved by initial morphological examination of the colonies in the plate (macroscopically) for colonial appearance, size, elevation, form, edge, consistency, colour, odour, opacity, haemolysis and pigmentation hence result was recorded.

The biochemical tests including catalase test, coagulase test, citrate utilization test, oxidase test, triple sugar iron (TSI) agar, urease test, sugar fermentation test, methyl red test and indole production test. Physiochemical identification were carried out as described by [8]

\section{RESULT}

A total number of twenty (20)“balangu" samples were aseptically collected from five (5) different retail outlets within Bauchi metropolis for bacteriological analysis. Ten (10) out of the total number taken were collected with spice (spiced) while ten others were not spiced (unspiced). From this study, considerable number of bacteria were isolated and identified in both the spiced and the unspiced "balangu" samples with fourteen (14) different species of bacteria isolated.

Table 1 shows the total aerobic plate count (cfu/g) for each of the twenty (20) "balangu" samples collected with their collection sites (aerobic plate count for both spiced and unspiced "balangu" samples)

Table 2 relates the mean aerobic plate count (cfu/g) for both spiced and unspiced "balangu" samples collected from five (5) different retail outlets within Bauchi metropolis.

The mean aerobic plate count in cfu/g for spiced "balangu" samples is $2.66 \times 10^{6}, 2.36 \times 10^{6}, 2.69 \mathrm{x}$ $10^{6}, 2.85 \times 10^{6}, 2.89 \times 10^{6}$, and for the unspiced "balangu" samples is $2.25 \times 10^{6}, 2.05 \times 10^{6}, 2.47 \times 10^{6}, 2.79 \times$ $10^{6}, 2.78 \times 10^{6}$, for Yelwa, GRA, Bayangari, Railway and Mudalawal motor park retail outlets respectively.

The bar chart in figure 2 shows Mudalawal motor part with the highest mean aerobic plate count (cfu/g) and GRA with the lowest for spiced "balangu" samples while Railway outlet gave the highest mean count also GRA still remain the lowest for the unspiced "balangu" samples.

Table 3, 4, 5, 6, and 7 shows the bacteria identified in both spiced and unspiced "balangu" samples which include Bacillus cereus, Staphylococcus aureus, Escherichia coli, Bacillus lentus, Bacillus alvis, Proteus vulgaris, Proteus mirabilis, Salmonella sp., Shigella sp., Citrobacter freundii, Streptococcus faecalis, Klebsiella pneumoniae, Enterobacter eurogenas and Micrococcus sp., hence elaborate morphological and biochemical properties of the bacteria isolates identified from the twenty (20)"balangu" samples collected from each of the five (5) retail outlets.

Table 8 relates the frequency of occurrence of each bacterium isolate in "balangu" samples collected from each retail outlet and the overall percentage of the occurrence of each bacterium isolated and identified in this experimental work.

TABLE 1: Total aerobic plate count (tapc) in (cfu/g) for twenty (20) "balangu" samples collected from the five $(5)$ retail outlets

\begin{tabular}{|c|c|c|c|c|c|}
\hline \multirow[t]{2}{*}{ Retail outlet } & \multicolumn{2}{|c|}{ Unspiced "balangu” samples } & \multicolumn{2}{|c|}{ Spiced "balangu" samples } & \multirow[t]{2}{*}{ no. Of samples } \\
\hline & Sample 1 & Sample 2 & Sample 1 & Sample 2 & \\
\hline Yelwa & $2.20 \times 10^{6}$ & $2.31 \times 10^{6}$ & $2.56 \times 10^{6}$ & $2.76 \times 10^{6}$ & 4 \\
\hline $\begin{array}{c}\text { Government reserve } \\
\text { area }\end{array}$ & $2.10 \times 10^{6}$ & $2.00 \times 10^{6}$ & $2.41 \times 10^{6}$ & $2.32 \times 10^{6}$ & 4 \\
\hline Bayangari & $2.23 \times 10^{6}$ & $2.72 \times 10^{6}$ & $2.57 \times 10^{6}$ & $2.81 \times 10^{6}$ & 4 \\
\hline Railway & $2.89 \times 10^{6}$ & $2.70 \times 10^{6}$ & $2.80 \times 10^{6}$ & $2.90 \times 10^{6}$ & 4 \\
\hline $\begin{array}{l}\text { Mudalawal } \\
\text { park }\end{array}$ & $2.76 \times 10^{6}$ & $2.81 \times 10^{6}$ & $2.87 \times 10^{6}$ & $2.92 \times 10^{6}$ & 4 \\
\hline \multicolumn{5}{|c|}{ Total number of samples collected } & 20 \\
\hline
\end{tabular}


Isolation and Identification of Bacteria Associated with Balangu (Roasted Meat Product)...

TABLE 2 : Mean aerobic plate count in (cfu/g) of both unspiced \& spiced "balangu" samples for the (5) retail outlet.

\begin{tabular}{|c|l|l|}
\hline & $\begin{array}{l}\text { Unspiced "balangu" } \\
\text { samples }\end{array}$ & $\begin{array}{l}\text { Spiced "balangu" } \\
\text { samples }\end{array}$ \\
\hline Retail outlet & $2.25 \times 10^{6}$ & $2.66 \times 10^{6}$ \\
\hline Government reserve area & & $2.36 \times 10^{6}$ \\
\hline Bayangari & $2.05 \times 10^{6}$ & $2.69 \times 10^{6}$ \\
\hline Railway & $2.47 \times 10^{6}$ & $2.85 \times 10^{6}$ \\
\hline Mudalawal park & & $2.89 \times 10^{6}$ \\
\hline
\end{tabular}

Figure 1 shows mean aerobic plate count (cfu/gram) of both unspiced and spiced "balangu" samples for the five (5) retail outlets 
Isolation and Identification of Bacteria Associated with Balangu (Roasted Meat Product)...

TABLE3 : Morphological \& biochemical properties of bacteria isolated in "balangu" samples collected from Yelwa retail outlet

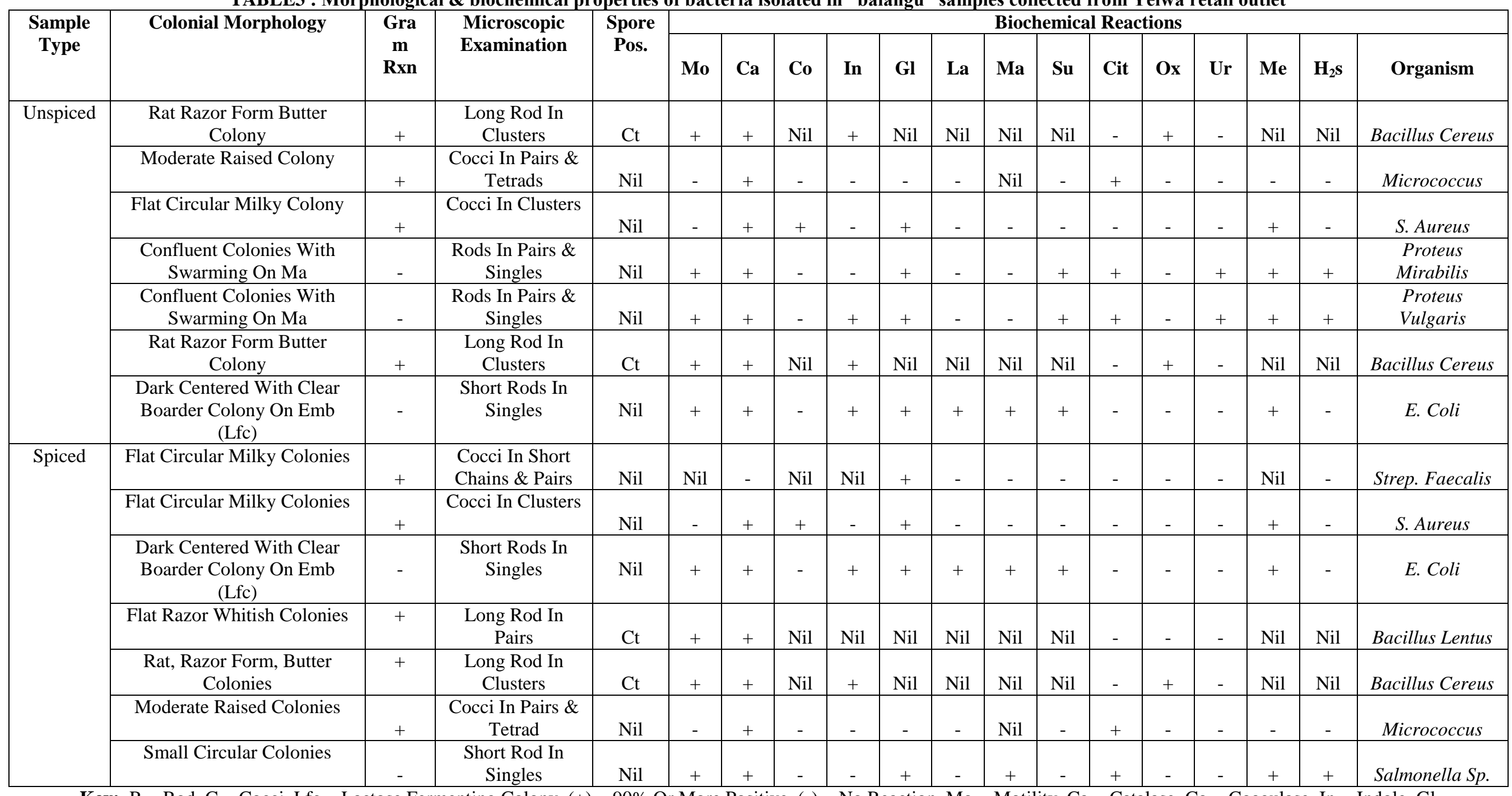

Key: $\mathrm{R}=$ Rod, $\mathrm{C}=$ Cocci, Lfc $=$ Lactose Fermenting Colony, $(+)=90 \%$ Or More Positive, $(-)=$ No Reaction, Mo $=$ Motility, Ca $=$ Catalase, Co $=$ Coagulase, In $=$ Indole, Gl $=$ Glucose, $\mathrm{La}=$ Lactose, $\mathrm{Ma}=$ Manitol, $\mathrm{Su}=$ Sucrose, $\mathrm{Ci}=$ Citrate, $\mathrm{Ox}=$ Oxidase, $\mathrm{Ur}=$ Urease, $\mathrm{Me}=$ Methyl Red, $\mathrm{H}_{2} \mathrm{~s}=$ Hydrogen Sulphide. 
Isolation and Identification of Bacteria Associated with Balangu (Roasted Meat Product)...

TABLE 4 : Morphological \& biochemical properties of bacteria isolated in "balangu" samples collected from GRA retail outlet

\begin{tabular}{|c|c|c|c|c|c|c|c|c|c|c|c|c|c|c|c|c|c|c|}
\hline \multirow{2}{*}{$\begin{array}{l}\text { Samples } \\
\text { Type }\end{array}$} & \multirow[t]{2}{*}{ Colonial Morphology } & \multirow{2}{*}{$\begin{array}{c}\text { Gram } \\
\text { Rnx }\end{array}$} & \multirow{2}{*}{$\begin{array}{l}\text { Microscopic } \\
\text { Examination }\end{array}$} & \multirow{2}{*}{$\begin{array}{l}\text { Spore } \\
\text { Pos. }\end{array}$} & \multicolumn{14}{|c|}{ Biochemical Reactions } \\
\hline & & & & & Mo & $\mathbf{C a}$ & Co & In & GI & La & Ma & Su & Cit & $\mathbf{O x}$ & Ur & Me & $\mathbf{H}_{2} \mathbf{S}$ & Organism \\
\hline \multirow[t]{3}{*}{ Unspiced } & Raise Circular Colonies & + & $\begin{array}{l}\text { Long Rods In } \\
\text { Clusters } \\
\end{array}$ & $\mathrm{Ct}$ & + & + & Nil & + & Nil & Nil & Nil & Nil & - & + & - & Nil & Nil & $\begin{array}{l}\text { Bacillus } \\
\text { Cereus }\end{array}$ \\
\hline & $\begin{array}{c}\text { Dark Centered With Clear } \\
\text { Boarder Colony On Emb } \\
\text { (Lfc) }\end{array}$ & - & $\begin{array}{l}\text { Short Rods In } \\
\text { Singles }\end{array}$ & Nil & + & + & - & + & + & + & + & + & - & - & - & + & - & E. Coli \\
\hline & Flat Circular Milky Colonies & + & Cocci In Clusters & Nil & - & + & + & - & + & - & - & - & - & - & - & + & - & S. Aureus \\
\hline \multirow[t]{5}{*}{ Spiced } & Flat Circular Milky Colonies & + & Cocci In Clusters & Nil & - & + & + & - & + & - & - & - & - & - & - & + & - & S. Aureus \\
\hline & $\begin{array}{c}\text { Raise Circular Colorless } \\
\text { Colonies }\end{array}$ & + & $\begin{array}{c}\text { Long Rods In } \\
\text { Clusters }\end{array}$ & $\mathrm{Ct}$ & + & + & Nil & + & Nil & Nil & Nil & Nil & - & + & - & Nil & Nil & $\begin{array}{c}\text { Bacillus } \\
\text { Cereus }\end{array}$ \\
\hline & $\begin{array}{c}\text { Raise Circular Colorless } \\
\text { Colonies }\end{array}$ & + & $\begin{array}{l}\text { Long Rods In } \\
\text { Singles }\end{array}$ & $\mathrm{Ct}$ & + & + & Nil & + & Nil & Nil & Nil & Nil & - & + & - & Nil & Nil & $\begin{array}{l}\text { Bacillus } \\
\text { Cereus }\end{array}$ \\
\hline & $\begin{array}{l}\text { Confluent Colonies With } \\
\text { Swarming On Ma }\end{array}$ & - & $\begin{array}{l}\text { Rods Pairs \& } \\
\text { Singles }\end{array}$ & Nil & + & + & - & - & + & - & - & + & + & - & + & + & + & $\begin{array}{c}\text { Proteus } \\
\text { Mirabilis }\end{array}$ \\
\hline & Moderate Raised Colony & + & $\begin{array}{l}\text { Cocci In Pairs \& } \\
\text { Tetrads }\end{array}$ & Nil & - & + & - & - & - & - & Nil & - & + & - & - & - & - & Micrococcus \\
\hline
\end{tabular}

Key: R = Rod, C = Cocci, Lfc = Lactose Fermenting Colony, $(+)=90 \%$ Or More Positive, $(-)=$ No Reaction, Mo $=$ Motility, Ca $=$ Catalase, Co = Coagulase, In = Indole, Gl $=$ Glucose, $\mathrm{La}=$ Lactose, $\mathrm{Ma}=$ Manitol, $\mathrm{Su}=$ Sucrose, $\mathrm{Ci}=$ Citrate, $\mathrm{Ox}=$ Oxidase, $\mathrm{Ur}=$ Urease, $\mathrm{Me}=\mathrm{Methyl}$ Red, $\mathrm{H}_{2} \mathrm{~s}=\mathrm{Hydrogen}$ Sulphide 
Isolation and Identification of Bacteria Associated with Balangu (Roasted Meat Product)...

TABLE 5 : Morphological \& biochemical properties of bacteria isolated in "balangu" samples collected from Bayangari retail outlet

\begin{tabular}{|c|c|c|c|c|c|c|c|c|c|c|c|c|c|c|c|c|c|c|}
\hline \multirow{2}{*}{$\begin{array}{l}\text { Samples } \\
\text { Type }\end{array}$} & \multirow[t]{2}{*}{ Colonial Morphology } & \multirow{2}{*}{$\begin{array}{c}\text { Gram } \\
\text { Rnx }\end{array}$} & \multirow{2}{*}{$\begin{array}{l}\text { Microscopic } \\
\text { Examination }\end{array}$} & \multirow{2}{*}{$\begin{array}{l}\text { Spore } \\
\text { Pos. }\end{array}$} & \multicolumn{14}{|c|}{ Biochemical Reactions } \\
\hline & & & & & Mo & $\mathbf{C a}$ & Co & In & Gl & $\mathbf{L a}$ & Ma & Su & Cit & $\mathbf{O x}$ & $\mathbf{U} \mathbf{r}$ & Me & $\mathbf{H}_{2} \mathbf{S}$ & Organism \\
\hline \multirow[t]{4}{*}{ Unspiced } & $\begin{array}{c}\text { Dark Centered With Clear } \\
\text { Boarder Colony On Emb } \\
\text { (Lfc) }\end{array}$ & - & $\begin{array}{l}\text { Short Rods In } \\
\text { Singles }\end{array}$ & Nil & + & + & - & + & + & + & + & $\mathrm{V}$ & - & - & - & + & - & E. Coli \\
\hline & $\begin{array}{c}\text { Flat Razor Whitish } \\
\text { Colonies }\end{array}$ & + & $\begin{array}{l}\text { Long Rods In } \\
\text { Pairs }\end{array}$ & $\mathrm{Ct}$ & - & + & Nil & + & Nil & Nil & Nil & Nil & - & Nil & + & Nil & Nil & Bacillus Alvis \\
\hline & $\begin{array}{c}\text { Flat Razor Whitish } \\
\text { Colonies }\end{array}$ & + & $\begin{array}{l}\text { Long Rods In } \\
\text { Scattered } \\
\end{array}$ & $\mathrm{Ct}$ & - & + & Nil & + & Nil & Nil & Nil & Nil & - & Nil & + & Nil & Nil & Bacillus Alvis \\
\hline & $\begin{array}{c}\text { Flat Circular Milky } \\
\text { Colonies } \\
\end{array}$ & + & $\begin{array}{l}\text { Cocci In Short } \\
\text { Chains } \\
\end{array}$ & Nil & Nil & - & Nil & Nil & + & - & - & - & - & - & - & Nil & - & $\begin{array}{c}\text { Strep. } \\
\text { Faecalis }\end{array}$ \\
\hline \multirow[t]{3}{*}{ Spiced } & $\begin{array}{c}\text { Flat Circular Milky } \\
\text { Colonies }\end{array}$ & + & Cocci In Clusters & Nil & - & + & + & - & + & - & - & - & - & - & - & + & - & $\begin{array}{c}\text { Staphylococc } \\
\text { us Aureus }\end{array}$ \\
\hline & $\begin{array}{c}\text { Confluent Colonies With } \\
\text { Swarming On Ma }\end{array}$ & - & $\begin{array}{c}\text { Rods In Pairs \& } \\
\text { Singles }\end{array}$ & Nil & + & + & - & - & + & - & - & + & + & - & + & + & + & $\begin{array}{c}\text { Proteus } \\
\text { Mirabilis }\end{array}$ \\
\hline & Small Circular Colonies & - & $\begin{array}{l}\text { Short Rods In } \\
\text { Singles }\end{array}$ & Nil & - & Nil & Nil & + & - & - & + & - & - & - & - & + & - & Shigella Sp. \\
\hline
\end{tabular}

Key: R = Rod, C = Cocci, Lfc = Lactose Fermenting Colony, (+) = 90\% Or More Positive, $(-)=$ No Reaction, Mo = Motility, Ca = Catalase, Co = Coagulase, In = Indole, Gl

$=$ Glucose, $\mathrm{La}=$ Lactose, $\mathrm{Ma}=$ Manitol, $\mathrm{Su}=$ Sucrose, $\mathrm{Ci}=$ Citrate, $\mathrm{Ox}=$ Oxidase, $\mathrm{Ur}=$ Urease, $\mathrm{Me}=\mathrm{Methyl}$ Red, $\mathrm{H}_{2} \mathrm{~s}=\mathrm{Hydrogen} \mathrm{Sulphide}$. 
Isolation and Identification of Bacteria Associated with Balangu (Roasted Meat Product)...

TABLE 6 : Morphological \& biochemical properties of bacteria isolated in "balangu" samples collected from Railway retail outlet

\begin{tabular}{|c|c|c|c|c|c|c|c|c|c|c|c|c|c|c|c|c|c|c|}
\hline \multirow{2}{*}{$\begin{array}{l}\text { Sample } \\
\text { Type }\end{array}$} & \multirow[t]{2}{*}{ Colonial morphology } & \multirow{2}{*}{$\begin{array}{c}\text { Gram } \\
\text { rnx }\end{array}$} & \multirow{2}{*}{$\begin{array}{l}\text { Microscopic } \\
\text { examination }\end{array}$} & \multirow{2}{*}{$\begin{array}{l}\text { Spore } \\
\text { pos. }\end{array}$} & \multicolumn{14}{|c|}{ Biochemical reactions } \\
\hline & & & & & $\begin{array}{c}\mathbf{M} \\
\mathbf{0}\end{array}$ & $\mathbf{C a}$ & Co & In & Gl & La & Ma & Su & Cit & $\mathbf{O x}$ & $\mathbf{U} \mathbf{r}$ & Me & $\mathbf{H}_{2} \mathrm{~S}$ & Organism \\
\hline \multirow[t]{6}{*}{ unspiced } & $\begin{array}{l}\text { Dark centered with clear boarder } \\
\text { colonies on EMB (LFC) }\end{array}$ & - & $\begin{array}{c}\text { Rods in pairs \& } \\
\text { singles }\end{array}$ & NIL & + & + & $\begin{array}{l}\text { NI } \\
\text { L }\end{array}$ & - & + & $\mathrm{V}$ & + & $\mathrm{V}$ & + & - & $\mathrm{V}$ & + & + & $\begin{array}{l}\text { Citrobacter } \\
\text { freundii }\end{array}$ \\
\hline & Flat razor whitish colonies & + & $\begin{array}{l}\text { Long rods in } \\
\text { clusters }\end{array}$ & $\mathrm{CT}$ & + & + & $\begin{array}{l}\text { NI } \\
\text { L }\end{array}$ & $\begin{array}{l}\text { NI } \\
\mathrm{L}\end{array}$ & $\begin{array}{l}\text { NI } \\
\text { L }\end{array}$ & $\begin{array}{c}\mathrm{NI} \\
\mathrm{L}\end{array}$ & NIL & $\begin{array}{l}\mathrm{NI} \\
\mathrm{L}\end{array}$ & - & - & - & $\begin{array}{l}\mathrm{NI} \\
\mathrm{L}\end{array}$ & NIL & $\begin{array}{c}\text { Bacillus } \\
\text { lentus }\end{array}$ \\
\hline & Raise circular colorless colonies & + & $\begin{array}{l}\text { Long rods in } \\
\text { clusters }\end{array}$ & CT & + & + & $\begin{array}{l}\mathrm{NI} \\
\mathrm{L}\end{array}$ & + & $\begin{array}{l}\mathrm{NI} \\
\mathrm{L}\end{array}$ & $\begin{array}{c}\mathrm{NI} \\
\mathrm{L}\end{array}$ & NIL & $\begin{array}{c}\mathrm{NI} \\
\mathrm{L}\end{array}$ & - & + & - & $\begin{array}{c}\mathrm{NI} \\
\mathrm{L}\end{array}$ & NIL & $\begin{array}{c}\text { Bacillus } \\
\text { cereus }\end{array}$ \\
\hline & $\begin{array}{l}\text { Confluent colonies with } \\
\text { swarming on MA }\end{array}$ & - & $\begin{array}{l}\text { Rod in pairs \& } \\
\text { singles }\end{array}$ & NIL & + & + & - & + & + & - & - & + & + & - & + & + & + & $\begin{array}{c}\text { Proteus } \\
\text { vulgaris }\end{array}$ \\
\hline & $\begin{array}{l}\text { Dark centered with clear boarder } \\
\text { colony on EMB (LFC) }\end{array}$ & - & $\begin{array}{l}\text { Short rods in } \\
\text { singles }\end{array}$ & NIL & + & + & - & + & + & + & + & + & - & - & - & + & - & E. coli \\
\hline & Rat, razors form, butter colonies & + & $\begin{array}{l}\text { Long rod in } \\
\text { singles }\end{array}$ & CT & + & + & $\begin{array}{c}\text { NI } \\
\mathrm{L}\end{array}$ & + & $\begin{array}{c}\mathrm{NI} \\
\mathrm{L}\end{array}$ & $\begin{array}{c}\text { NI } \\
\text { L }\end{array}$ & NIL & $\begin{array}{c}\mathrm{NI} \\
\mathrm{L}\end{array}$ & - & + & - & $\begin{array}{c}\mathrm{NI} \\
\mathrm{L}\end{array}$ & NIL & $\begin{array}{l}\text { Bacillus } \\
\text { cereus }\end{array}$ \\
\hline \multirow[t]{7}{*}{ Spiced } & $\begin{array}{c}\text { Dark centered with clear boarder } \\
\text { colony on EMB (LFC) }\end{array}$ & - & $\begin{array}{c}\text { Rods in pairs \& } \\
\text { singles }\end{array}$ & NIL & + & + & $\begin{array}{c}\mathrm{NI} \\
\mathrm{L}\end{array}$ & - & + & + & + & + & + & - & + & + & + & $\begin{array}{l}\text { Citrobacter } \\
\text { freundii }\end{array}$ \\
\hline & Small circular colonies & - & $\begin{array}{l}\text { Short rod in } \\
\text { single }\end{array}$ & NIL & - & NIL & $\begin{array}{l}\text { NI } \\
\text { L }\end{array}$ & + & - & - & + & - & - & - & - & + & - & Shigella sp. \\
\hline & Raise circular milky colonies & + & $\begin{array}{c}\text { Long rod in } \\
\text { cluster }\end{array}$ & $\mathrm{CT}$ & + & + & $\begin{array}{c}\text { NI } \\
\text { L }\end{array}$ & + & $\begin{array}{l}\text { NI } \\
\text { L }\end{array}$ & $\begin{array}{c}\mathrm{NI} \\
\mathrm{L}\end{array}$ & NIL & $\begin{array}{c}\text { NI } \\
\text { L }\end{array}$ & - & + & - & $\begin{array}{c}\mathrm{NI} \\
\mathrm{L}\end{array}$ & NIL & $\begin{array}{c}\text { Bacillus } \\
\text { cereus }\end{array}$ \\
\hline & Flat circular milky colonies & + & $\begin{array}{l}\text { Cocci in } \\
\text { clusters }\end{array}$ & NIL & - & + & + & - & + & - & - & - & - & - & - & + & - & $\begin{array}{c}\text { Staphylococc } \\
\text { us aureus }\end{array}$ \\
\hline & Flat circular milky colonies & + & $\begin{array}{l}\text { Cocci in short } \\
\text { chains \& pairs }\end{array}$ & NIL & + & - & $\begin{array}{l}\mathrm{NI} \\
\mathrm{L}\end{array}$ & $\begin{array}{c}\mathrm{NI} \\
\mathrm{L}\end{array}$ & + & - & - & - & - & - & - & $\begin{array}{c}\mathrm{NI} \\
\mathrm{L}\end{array}$ & - & $\begin{array}{c}\text { Strep. } \\
\text { faecalis }\end{array}$ \\
\hline & $\begin{array}{c}\text { Dark centered with clear boarder } \\
\text { colony on EMB (LFC) }\end{array}$ & - & $\begin{array}{l}\text { Long rods in } \\
\text { singles }\end{array}$ & NIL & - & + & - & - & + & + & + & + & + & - & + & + & - & $\begin{array}{l}\text { Klebsiella } \\
\text { pneumonia }\end{array}$ \\
\hline & Moderate raised colony & + & $\begin{array}{c}\text { Cocci in pairs \& } \\
\text { tetrads }\end{array}$ & NIL & - & + & - & - & - & - & NIL & - & + & - & - & - & - & Micrococcus \\
\hline
\end{tabular}

Key: R = Rod, C = Cocci, Lfc = Lactose Fermenting Colony, $(+)=90 \%$ Or More Positive, $(-)=$ No Reaction, Mo = Motility, Ca $=$ Catalase, Co = Coagulase, In = Indole, Gl $=$ Glucose, $\mathrm{La}=$ Lactose, $\mathrm{Ma}=$ Manitol, $\mathrm{Su}=$ Sucrose, $\mathrm{Ci}=$ Citrate, $\mathrm{Ox}=$ Oxidase, $\mathrm{Ur}=$ Urease, $\mathrm{Me}=$ Methyl Red, $\mathrm{H}_{2} \mathrm{~s}=$ Hydrogen Sulphide. 
Isolation and Identification of Bacteria Associated with Balangu (Roasted Meat Product)...

TABLE 7 : Morphological \& biochemical properties of bacteria isolated in "balangu" samples collected from Mudalawal motor park outlet

\begin{tabular}{|c|c|c|c|c|c|c|c|c|c|c|c|c|c|c|c|c|c|c|}
\hline \multirow{2}{*}{ Samples } & \multirow[t]{2}{*}{ Colonial Morphology } & \multirow{2}{*}{$\begin{array}{l}\text { Gram } \\
\text { Rnx }\end{array}$} & \multirow{2}{*}{$\begin{array}{l}\text { Microscopic } \\
\text { Examination }\end{array}$} & \multirow{2}{*}{$\begin{array}{l}\text { Spore } \\
\text { Pos. }\end{array}$} & \multicolumn{14}{|c|}{ Biochemical Reactions } \\
\hline & & & & & Mo & $\mathrm{Ca}$ & Co & In & Gl & La & Ma & $\mathrm{Su}$ & Cit & $\mathbf{O x}$ & $\mathbf{U r}$ & Me & $\mathbf{H}_{2} \mathbf{S}$ & Organism \\
\hline \multirow[t]{5}{*}{$\begin{array}{l}\text { Unspice } \\
\mathrm{d}\end{array}$} & $\begin{array}{ll}\text { Confluent Colonies } & \text { With } \\
\text { Swarming On Ma } & \\
\end{array}$ & - & $\begin{array}{l}\text { Rods In Pairs \& } \\
\text { Singles }\end{array}$ & Nil & + & + & - & - & + & - & - & + & + & - & + & + & + & $\begin{array}{l}\text { Proteus } \\
\text { Mirabilis }\end{array}$ \\
\hline & Flat Razor Whitish Colonies & + & $\begin{array}{l}\text { Long Rods In } \\
\text { Cluster }\end{array}$ & $\mathrm{Ct}$ & - & + & Nil & + & Nil & Nil & Nil & Nil & - & Nil & + & Nil & Nil & $\begin{array}{l}\text { Bacillus } \\
\text { Alvis }\end{array}$ \\
\hline & $\begin{array}{l}\text { Dark Centered With Clear Boarder } \\
\text { Colony On Emb (Lfc) }\end{array}$ & - & $\begin{array}{lll}\text { Long } & \text { Rod } & \text { In } \\
\text { Singles } & & \\
\end{array}$ & Nil & - & + & - & - & + & + & + & + & + & - & + & + & - & $\begin{array}{l}\text { Klebsiella } \\
\text { Pneumonia }\end{array}$ \\
\hline & Flat Razor Whitish Colonies & + & $\begin{array}{l}\text { Long Rods In } \\
\text { Clusters }\end{array}$ & $\mathrm{Ct}$ & - & + & Nil & + & Nil & Nil & Nil & Nil & - & Nil & + & Nil & Nil & $\begin{array}{l}\text { Bacillus } \\
\text { Alvis }\end{array}$ \\
\hline & $\begin{array}{l}\text { Dark Centered With Clear Boarder } \\
\text { Colony On Emb (Lfc) }\end{array}$ & - & $\begin{array}{lll}\text { Short } & \text { Rods } & \text { In } \\
\text { Singles } & & \\
\end{array}$ & Nil & + & + & - & + & + & + & + & + & - & - & - & + & - & E. Coli \\
\hline \multirow[t]{8}{*}{ Spiced } & $\begin{array}{l}\text { Dark Centered With Clear Boarder } \\
\text { Colony On Emb (Lfc) }\end{array}$ & - & Rods In Singles & Nil & + & + & Nil & - & + & + & + & + & + & - & - & - & - & $\begin{array}{l}\text { Enterobacter } \\
\text { Eurogenas }\end{array}$ \\
\hline & Flat Circular Milky Colonies & + & Cocci In Clusters & Nil & - & + & + & - & + & - & - & - & - & - & - & + & - & $\begin{array}{l}\text { Staphylococc } \\
\text { us Aureus }\end{array}$ \\
\hline & Raise Circular Milky Colonies & + & $\begin{array}{l}\text { Long Rods In } \\
\text { Clusters }\end{array}$ & $\mathrm{Ct}$ & + & + & Nil & + & Nil & Nil & Nil & Nil & - & + & - & Nil & Nil & $\begin{array}{l}\text { Bacillus } \\
\text { Cereus }\end{array}$ \\
\hline & $\begin{array}{l}\text { Dark Centered With Clear Boarder } \\
\text { Colony On Emb (Lfc) }\end{array}$ & - & Rods In Singles & Nil & + & + & Nil & - & + & + & + & + & + & - & - & - & - & $\begin{array}{l}\text { Enterobacter } \\
\text { Eurogenas }\end{array}$ \\
\hline & $\begin{array}{ll}\text { Confluent Colonies } & \text { With } \\
\text { Swarming On Ma } & \\
\end{array}$ & - & $\begin{array}{l}\text { Rods In Pairs \& } \\
\text { Singles }\end{array}$ & Nil & + & + & - & + & + & - & - & + & + & - & + & + & + & $\begin{array}{l}\text { Proteus } \\
\text { Vulgaris }\end{array}$ \\
\hline & Flat Circular Milky Colonies & + & $\begin{array}{l}\text { Cocci In Short } \\
\text { Chains \& Pairs }\end{array}$ & Nil & Nil & - & Nil & Nil & + & - & - & - & - & - & - & Nil & - & $\begin{array}{l}\text { Strep. } \\
\text { Faecalis }\end{array}$ \\
\hline & Small Circular Colonies & - & $\begin{array}{lll}\text { Short } & \text { Rod } & \text { In } \\
\text { Single } & & \\
\end{array}$ & Nil & - & Nil & Nil & + & - & - & + & - & - & - & - & + & - & Shigella Sp. \\
\hline & Small Circular Colonies & - & $\begin{array}{lll}\text { Short } & \text { Rod } & \text { In } \\
\text { Singles } & & \end{array}$ & Nil & + & + & - & - & + & - & + & - & + & - & - & + & + & $\begin{array}{l}\text { Salmonella } \\
\text { Sp. }\end{array}$ \\
\hline
\end{tabular}

Key: R = Rod, C = Cocci, Lfc = Lactose Fermenting Colony, $(+)=90 \%$ Or More Positive, $(-)=$ No Reaction, Mo = Motility, Ca = Catalase, Co = Coagulase, In = Indole, Gl $=$ Glucose, $\mathrm{La}=$ Lactose, $\mathrm{Ma}=$ Manitol, $\mathrm{Su}=$ Sucrose, $\mathrm{Ci}=$ Citrate, $\mathrm{Ox}=$ Oxidase, $\mathrm{Ur}=$ Urease, $\mathrm{Me}=\mathrm{Methyl} \mathrm{Red}, \mathrm{H}_{2} \mathrm{~s}=\mathrm{Hydrogen}$ Sulphide . 
Isolation and Identification of Bacteria Associated with Balangu (Roasted Meat Product)...

TABLE 8 : Frequency of occurrence of bacterial isolates

\begin{tabular}{llllllll}
\hline Organism & \multicolumn{7}{c}{ Number of occurrence } \\
\cline { 2 - 8 } & Yelwa & GRA & Bayangari & Railway & $\begin{array}{l}\text { M/Lawal } \\
\text { park }\end{array}$ & Total & Percentage \\
\hline Bacillus cereus & 3 & 3 & 1 & 3 & 1 & 11 & 19.6 \\
Staphylococcus aureus & 2 & 2 & 1 & 1 & 1 & 7 & 12.5 \\
Escherichia coli & 2 & 1 & 1 & 1 & 1 & 6 & 10.7 \\
Bacillus lentus & 1 & Nil & Nil & 1 & Nil & 2 & 3.5 \\
Bacillus alvis & Nil & Nil & 2 & Nil & 2 & 4 & 7.1 \\
Proteus vulgaris & 1 & Nil & Nil & 1 & 1 & 3 & 5.4 \\
Proteus mirabilis & 1 & 1 & 1 & Nil & 1 & 4 & 7.1 \\
Salmonella sp & 1 & Nil & Nil & Nil & 1 & 2 & 3.5 \\
Shigella sp & Nil & Nil & 1 & 1 & 1 & 3 & 5.4 \\
Citrobacter freundii & Nil & Nil & Nil & 2 & Nil & 2 & 3.5 \\
Streptococcus faecalis & 1 & Nil & 1 & 1 & 1 & 4 & 7.1 \\
Klebsiella pneumoniae & Nil & Nil & Nil & 1 & 1 & 2 & 3.5 \\
Enterobacter eurogenase & Nil & Nil & Nil & Nil & 2 & 2 & 3.5 \\
Micrococcus & 2 & 1 & Nil & 1 & Nil & 4 & 7.1 \\
& \multicolumn{7}{c}{ Total } \\
\hline
\end{tabular}

\section{DISCUSSION}

This significant rise in bacterial load in Railway and Mudalawal retail outlets compared to others for both spiced and unspiced "balangu" samples could be as a result of poor processing method, poor hygiene practice, improper and unhygienic handling of the meat product, bad sanitation operations and use of unclean formites and utensils. [4]stated that one of the major sources of contamination arises from the handlers during preparation and display of meet product for sale. However an important factor which significantly contributes to the great increase in the count is the location of the retail outlet which are basically motor parks or railway station where "balangu" can be easily contaminated by aerial spores or bacterial spores carried in the air and several other insects, such as flies, which are uncountable at such sites. [9] states that insects such as flies can cause contamination by continuous contact with the product, so also dust particles from heavily contaminated atmospheres around market places and motor parks.

From Table 2, the mean aerobic plate count for spiced "balangu" samples is higher when compared to that of the unspiced "balangu" samples for all the retail outlets which may be due to the additional contamination arising from the spices used, or poor handling of the spices during preparation. Contamination of meat product could arise from spice and use of contaminated equipment/ utensils, and that spice may also be heavily loaded with microorganism which served as a good source of contamination of meat products[11].

Generally, the increase level of bacterial load observed in this study could also be asa result of contamination arising from slaughter procedures (during bleeding, skinning and handling). [11] stated that contamination occur from various sources notably the present states of abattoir, slaughter house, slaughter procedure and practices may result in heavily contaminated meat.

Another source of contamination could be the retailer themselves, as most are dressed in filthy clothes, also the product stand and location plays a significant role as most samples analysed were sold in market places, along road sides, near refuse dump and Motor Park where meat products are easily contaminated directly or indirectly. Other sources of contamination are packaging materials, which are often sourced from nooks and corners.

Percentage of occurrence of bacterial isolated in relation to all the retail outlets is highest for Bacillus cereus with $19.6 \%$ which may be due to contamination from aerial spores carried in the air.Staphylococcus aureus was also found in the "balangu" meat product due to poor personal hygiene.S. aureus are found in human nose, throats, skin and 50-60\% of normal people are carriers thus shows $12.5 \%$ occurrence while Bacillus lentus, Salmonella sp, Shigella sp, Citrobacter freundii, Klebsiella pneumoniae and Enterococcus eurogenase shows the lowest percentage of occurrence of $3.5 \%$.

Salmonella sppcan survive improper heating of "balangu" meat product during processing. [1] stated that the presence of Salmonella spas contaminant could be attributed to inadequate heating of meat product during its preparation.

It is important to note that Streptococcus faecalis, Staphylococcus aureus, Bacillus sp, Micrococcus are mostly isolated in spiced "balangu" samples thus confirmed contamination resulting from spice therefore most bacteria isolated in "balangu" are of public health importance causing food borne diseases, food poisoning and food intoxication (e.g. Staphyloenterotoxemia), dysentery, travellers' diarrhoea, abdominal disorder and pains, 
Isolation and Identification of Bacteria Associated with Balangu (Roasted Meat Product)...

sore throats, mild fever and lots more while other are of no public health significance but signifies poor hygiene during processing and handling of meat products [1]

\section{CONCLUSION}

Some bacteria isolated and identified in the "balangu" meat product are of public health importance thus their presence in such meat product continues to be considered as major causes of gastro-intestinal disorders, food poisoning and food borne diseases.

\section{RECOMMENDATION}

1. National Agency for Food and Drugs Administration and Control should particularly regulate or set up a local body nationwide to monitor the production/processing, site of production (location), packaging of "balangu" and other meat products in order to reduce health hazard and guarantee good health for the nation.

2. There is need for establishment of national legislation that would restrict sales of "balangu" and other meat product in strategic locations to reduce the level of contamination of such meat products.

3. Customers should insist on adequate reheating of the "balangu" and any other meat product before purchase to ensure destruction of vegetative cells.

4. Proper meat inspection, screening and eradication of sick and unhealthy animals should be strictly adhered to hence abattoir construction and its operation should be able to limit or control excess contamination of raw meat.

5. customers should also insist on using sterile foil paper or polythene bags for packaging the "balangu" (roasted meat product) when purchased and retailers should avoid the use of cement paper and news print for they might be heavily contaminated.

6. Adequate measures and proper hygiene should be employed during the production of spice thus spice should be devoid of potential microbial contaminant.

7. The need for periodic screening of producers and retailers for convalescent carriers of micro-organism cannot be over emphasized.

8. There exist need to educate "balangu" producers or retailers on sanitation, personal hygiene and its relevance to food production.

9. Public health education programme is of good necessity to enlighten the general public about the health implication of consuming contaminated meat product..

\section{REFERENCE}

1). M.R. Adams and M.O. Moss. Food microbiology. The Royal Society of Chemistry, Thomas Graham house, Service Park, Cambridge, UK. 1999, 192-202.

2). I.Okala and A.Reedi. A Comprehensive Approach Africana Publishers Limited-Nigeria, 2001, 33-35.

3). I.A. Ikeme. Meat Science and Technology. A comprehensive approach. (Africana - FEP publishers Ltd. Onitsha, Nigeria 1990).

4). Baird-Parker A.C. Baird-parker (1993). Food and microbiological risks. Fred GriffithReview lecture In: Journal of Microbiology (1994) 140:687-695

5). J.M.Jay. Modern Food Microbiology. $6^{\text {th }}$ Edition. Aspen Publishers Inc. Gaithersburg. Maryland, 2000, 60-89.

6). A.I. Yunusa. Curing and Smoking Meat for home food preservation In Meat Science press, 2000, 175175.

7). M.J. James. Modern Food Microbiology $3^{\text {rd }}$ Edition (M.C. Tata Graw Hill Publishers Co. Ltd. 2001, 110-125).

8). U.A. Luyet2000. Bacterial Causing Beef Spoilage in a Meat shop In Ibadan, Nigeria. Journal of Microbiology. $1^{\text {st }}$ Edition, 2000,150-155

9). Monica and Chessbrough (2000). District laboratory practice in tropical courtiers.Part 2, Cambridge press UK, 2000.

10). S. A.Okughaba Microbiological safety and stability of Intermediate FrlOiStUffé beef. M. Sc. Thesis University of Nigeria Nsukka. 1986

11). U.J. Umoh Critical control points of beef products. http:/www.THIS DAY.online.com 